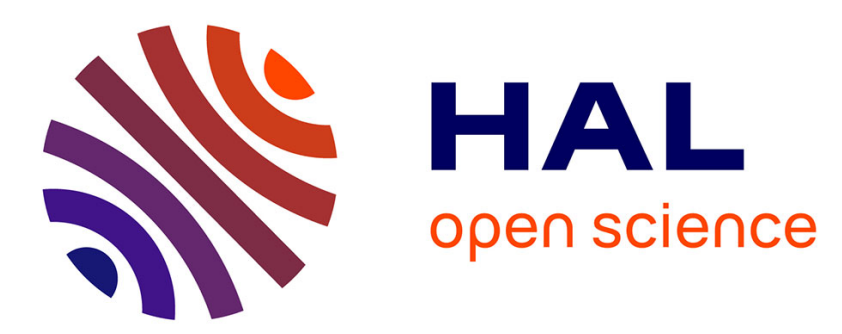

\title{
Maternités précoces: profils sociodémographiques de 220 mères adolescentes en Seine-Saint-Denis
}

\author{
Louise Genest, Hélène Decroix, Daniel Rotten, Laurence Simmat-Durand
}

\section{To cite this version:}

Louise Genest, Hélène Decroix, Daniel Rotten, Laurence Simmat-Durand. Maternités précoces : profils sociodémographiques de 220 mères adolescentes en Seine-Saint-Denis. Journal de Gynécologie Obstétrique et Biologie de la Reproduction, 2014, 43 (5), pp.351-360. 10.1016/j.jgyn.2013.03.009 . inserm-00818831

\section{HAL Id: inserm-00818831 https://www.hal.inserm.fr/inserm-00818831}

Submitted on 29 Apr 2013

HAL is a multi-disciplinary open access archive for the deposit and dissemination of scientific research documents, whether they are published or not. The documents may come from teaching and research institutions in France or abroad, or from public or private research centers.
L'archive ouverte pluridisciplinaire HAL, est destinée au dépôt et à la diffusion de documents scientifiques de niveau recherche, publiés ou non, émanant des établissements d'enseignement et de recherche français ou étrangers, des laboratoires publics ou privés. 
Résumé. Objectif : Dans le cadre de grossesses chez des adolescentes, il est souvent constaté une grande vulnérabilité et un suivi de grossesse de moindre qualité. Cette enquête vise à établir des profils de jeunes femmes et à évaluer ce suivi. Méthodes : Étude rétrospective (2007-2011) de 220 accouchements de femmes de 12 à 17 ans. Résultats : Le suivi de grossesse chez ces adolescentes reste globalement inférieur en qualité aux moyennes nationales françaises, mais des différences significatives sont observées lorsque l'on prend en compte des éléments sociodémographiques. Trois profils apparaissent : 66 adolescentes (groupe A) déclarent une surveillance de grossesse chaotique, avec une situation sociale, professionnelle, familiale peu renseignée. 102 adolescentes (groupe B) présentent un suivi de meilleure qualité mais la grossesse est très souvent non désirée avec une situation sociale instable. Enfin 52 adolescentes (groupe C) présentent un suivi plutôt de bonne qualité avec un investissement de la grossesse légèrement plus affirmé. Conclusion Tenir compte des conditions de vie de ces adolescentes est essentielle pour étudier les maternités précoces et pallier les vulnérabilités propres à cette population fragile. Un repérage des facteurs favorisant une surveillance de grossesse optimale chez ces jeunes femmes est mis en parallèle avec une réduction des risques obstétricaux et sociaux.

Mots clefs : maternité précoce, adolescence, suivi de grossesse. 
Summary. Objective: As part of teenage pregnancies, it is often found a vulnerability and a pregnancy follow-up of poorer quality. This investigation aims to establish profiles of young women and to assess what followed. Methods: A retrospective study (2007-2011) of 220 births to women aged 12 to 17 . Results: The monitoring of these teenage pregnancies remains generally lower than France national averages, but significant differences are observed when one takes into account the socio-demographic factors. Three profiles appear: 66 teenagers (group A) reported a chaotic pregnancy monitoring, with a social, professional, family somewhat knowledgeable. 102 teenagers (group B) have a better follow-up but pregnancy is often unwanted with an unstable social situation. Finally 52 teenagers (group C) have a rather good quality monitoring with a more assertive investment of pregnancy. Conclusion To consider the lives of these young women is essential to study early motherhood and mitigate the vulnerabilities of this fragile population. An identification of factors favoring an optimal monitoring of pregnancy among young women is paralleled with reduced obstetric and social risks.

Keywords: early motherhood, teenage years, pregnancy monitoring. 
Introduction : La tendance observée en France entre 1980 et 2000 d’un recours plus fréquent à l'Interruption Volontaire de Grossesse (IVG) en cas de grossesse chez les mineures (de 1/3 à 2/3 des conceptions) s'est encore accentuée aujourd'hui. Les IVG représentent $75 \%$ des conceptions de 2011 chez les jeunes femmes de moins de 18 ans (chaque année environ 4500 naissances sont observées contre 11000 en 1980) [1-5]. Les maternités adolescentes restent donc un phénomène relativement modéré et en diminution constante depuis quelques décennies. Le département de la Seine saint Denis est le plus fécond de France avec en moyenne 2,38 enfants par femme. Sur 2954 naissances en Ile-de-France en 2010 chez les moins de vingt ans, les données statistiques récentes font état de 627 naissances uniquement sur le département de la Seine-Saint-Denis. Le centre hospitalier de Saint-Denis est l’hôpital qui a vu naître le plus d'enfants issus de jeunes femmes de moins de 20 ans, soit 134 naissances sur l'année 2010. Parmi celles-ci notre enquête nous a permis d'identifier 45 mineures (Cf supra méthode). Il est très difficile d’obtenir des données chiffrées sur le nombre de grossesses chez les moins de 18 ans. Très souvent les découpages statistiques incluent des jeunes femmes de 19 et 20 ans, et c’est notamment le cas pour notre région d'étude [6]. Au niveau international, il existe évidemment des disparités mais dans la plupart des pays occidentaux, le nombre des naissances adolescentes tend à se réduire. Les taux les plus bas (5 à 7 naissances pour 1000 adolescentes de moins de 20 ans) se situent en Italie, en Suisse, aux Pays Bas, en Suède et en Slovénie. La France, l'Espagne et le Danemark occupent une position moyenne en Europe (autour de $11 \%$ ). Les taux les plus élevés sont ceux de la Roumanie (37\%o) et du Royaume-Uni (27 \%o) [7-9].

La grossesse chez l'adolescente en France est considérée comme une situation particulière, dérangeante, qui soulève des problèmes médicaux et sociaux, posant la question de la contraception et celle de sa capacité à être mère. Notre intérêt particulier pour les jeunes mères s'appuie sur de nombreuses études nous indiquant qu'elles forment une population 
cumulant plusieurs facteurs de vulnérabilité. En effet, la littérature tend à démontrer que les grossesses chez les adolescentes sont caractérisées essentiellement par un risque majeur d'accouchement prématuré et la naissance d'enfants de faible poids [10-15]. Ce phénomène s'expliquerait par l'immaturité biologique de ces jeunes filles (leur corps et la capacité à porter la vie n'est pas physiologiquement mature) et le manque de suivi médical au moment de leur grossesse. Selon le chercheur A. Khashan, la majorité de ces adolescentes a découvert sa grossesse tardivement et n'a pas effectué les examens prénataux nécessaires au bon déroulement de l'accouchement [10]. Cependant, l'incidence de ces risques s’infléchit sous l'influence d'un suivi optimal de la grossesse. D'autre part, certains facteurs socio démographiques récurrents, comme l'arrêt précoce de la scolarisation, l’absence d’activité professionnelle ou de relations familiales et sociales stables, semblent être inhérents aux mères adolescentes. Ces facteurs influent notablement sur le nombre de visites prénatales et la qualité du suivi obstétrical et périnatal. La présente enquête vise à présenter les caractéristiques sociodémographiques d'une population de mères adolescentes dans une étude rétrospective de 2007 à 2011 à l'hôpital Delafontaine (Saint-Denis, département de la SeineSaint-Denis, Ile-de-France). Elle cherchera également à définir des profils sociaux à partir de cette population et à évaluer la prise en charge obstétricale de ces jeunes femmes.

\section{Méthodologie et population étudiée.}

1) Le premier objectif de cette recherche quantitative a été tout d'abord de présenter une description la plus complète possible d'une population de jeunes femmes mineures ayant accouché au sein de la maternité du centre hospitalier de Saint-Denis (Hôpital Delafontaine) sur une durée de 5 années (1 janvier 2007 - 31 décembre 2011). L’ensemble de cette étude a été réalisée au sein même de ce service pendant un peu plus d'une année (de décembre 2010 à janvier 2012). 
À partir des cahiers de naissances utilisés dans le service de la maternité, nous avons pu déterminer le nombre de jeunes femmes âgées de moins de 18 ans ayant donné naissance à un enfant, vivant ou non (Mort Fœtale In Utero -MFIU- incluses), au sein de cet hôpital pendant ces années (nous n’avons pas tenu compte des IVG dans le cadre de cette recherche). Ainsi, 220 dossiers de femmes âgées de moins de 18 ans au jour de leur début de suivi de grossesse à la maternité ont été analysés de manière rétrospective : soit une quarantaine de grossesses par an. Sur la période de recueil, 15132 naissances ont été enregistrées à l’hôpital Delafontaine, l'un des plus importants centres hospitaliers du département de la Seine-Saint-Denis; disposant d’une maternité de type III [16]. Les dossiers obstétricaux des jeunes femmes ont servi de base de recueil à partir des questions pré-imprimées et surtout des notes des soignants en marge du dossier. La base de données renseignée comportait 158 variables décrivant les conditions de vie de l'adolescente, ses comportements (consommation de tabac et contraception), son passé obstétrical, le suivi de sa grossesse (nombre d’échographies et de consultations), les conditions de l'accouchement, les caractéristiques du nouveau-né, des données sur le père de l'enfant. Une variable score «de vulnérabilité » a été créée pour évaluer le nombre d’éléments psychosociaux jugés préoccupants par l’équipe obstétricale pour chaque jeune mère mineure : le logement de la jeune mère pendant la grossesse, l’existence de violences subies, des antécédents de placements ou mesures éducatives, un rejet familial à l'annonce de la grossesse, la qualité de la relation mère/fille, la situation conjugale et enfin la parité. Le score est ainsi compris entre 0 et 9 pour chaque femme. Seront considérées comme vulnérables les adolescentes obtenant un score supérieur ou égal à 2. Les traitements statistiques ont été réalisés avec le logiciel Modalisa6 (Kynos, Paris, France) et le test du Khi2 a été utilisé pour comparer les effectifs.

2) Notre second objectif de recherche consistait à créer une typologie des profils de jeunes mères, construite à partir de variables sociodémographiques significativement associées à la 
qualité du suivi de grossesse (soit: l’âge de la mère, l’année d'accouchement, le niveau d’éducation, l’activité professionnelle, la situation conjugale, la couverture sociale, l’origine ethnique, le logement, la prévision de la grossesse et son investissement). Une analyse factorielle des correspondances (AFC) a été utilisée pour mesurer les distances entre modalités. Dans Modalisa6, les classifications automatiques sont construites sur la base des distances entre individus sur les cartes d'AFC, le logiciel permet ensuite de créer une question fermée à partir des typologies : chaque profil présenté (A, B ou C) correspond alors à un type. 3) Enfin, notre dernier objectif a été d’évaluer les modalités de prise en charge de ces jeunes femmes en tenant compte des profils sociodémographiques créés antérieurement. La prise en charge a été modifiée au cours des années d’observation. En effet, une consultation spécialisée pour les jeunes femmes mineures enceintes a été mise en place depuis 2010. Entourée d’une équipe pluridisciplinaire, les jeunes femmes sont suivies très régulièrement et rencontrent successivement médecins, sages-femmes, puéricultrices, psychologues, assistantes sociales pour préparer la venue de leur enfant ; suivi qui peut se prolonger en suite de couches si les adolescentes le désirent. Ce travail en équipe a nettement influencé la qualité et la précision des données recueillies dans les dossiers qui se sont améliorées ces dernières années. Par contre les données recueillies sur les consommations de substances sont restées extrêmement lacunaires et imprécises, ne nous permettant pas de les exploiter, ni de les mentionner.

Enfin précisons qu'une des variables de notre questionnaire concerne l'entretien prénatal précoce. Nous sommes partis du principe que toute les adolescentes qui ont débuté précocement la surveillance de leur grossesse (par exemple en PMI) ont bénéficié de cet entretien prénatal. Pour celles qui sont arrivées en maternité au 6e mois de leur grossesse, et qui n’en n’avaient pas bénéficié, le médecin référent le réalisait de manière systématique. 
Ainsi, seules les adolescentes débutant un suivi de grossesse très tardif voire sans suivi n’en ont pas bénéficié.

\section{Résultats : Description de la population étudiée.}

Les adolescentes sont âgées en moyenne de 16 ans. La plus jeune mère est âgée de 12 ans et la plus «vieille » de 17 ans (Tab1). Majoritairement d’origine étrangère, seules 21 \% des jeunes filles sont originaires de France métropolitaine et $10 \%$ des DOM-TOM, un tiers est né en Afrique et essentiellement au Mali, Cap Vert et Congo, et $11 \%$ dans une autre région. Dans une grande majorité, les adolescentes sont déscolarisées au moment de l'enquête. Seules 39 \% d'entre elles déclarent être encore à l'école ou envisagent de poursuivre leurs études après la naissance de l'enfant et seulement $2 \%$ déclarent occuper un emploi. $51 \%$ de notre effectif s’est déclaré célibataire mais certaines peuvent avoir un compagnon sans vie commune. 69 \% des adolescentes étudiées vivent chez leurs parents au moment de la grossesse, pourcentage stable en fin de grossesse. Parmi celles-ci $28 \%$ des adolescentes ont déclaré vivre dans une caravane, au moment de leur grossesse comme après leur accouchement, ce qui correspond aux jeunes filles originaire de l'Europe de l'Est (Roumanie essentiellement).

16 \% des adolescentes de notre étude rétrospective, ont-elles-mêmes vécu un placement, soit en famille d'accueil, soit en foyer de l'Aide Sociale à l'Enfance (ASE). 7 \% des jeunes mères ont également été mises à la porte par leur famille au moment de l'annonce de la grossesse. 14 \% ont déclaré avoir subi des violences physiques au cours de leur vie : plus de la moitié décrivent des violences familiales régulières (tante, oncle, beau-père /belle-mère et parents) mais aussi des violences de la part de leur conjoint. 7 jeunes filles ont déclaré avoir été violées. Parmi celles-ci, 5 ont souhaité garder leur enfant et 2 ont choisi une interruption médicale de grossesse. 4 adolescentes ont accouché sous X. Enfin le score de vulnérabilité révèle que 59 \% de jeunes filles de notre étude rétrospective cumulent au minimum un facteur 
de vulnérabilité pendant leur grossesse (Cf méthode). En ce qui concerne les pères de ces enfants, ils sont âgés en moyenne de 22 ans (écart-type 5,4 ans) mais nous observons un taux de non réponse de 20 \%, notamment lié au fait que les équipes soignantes s’intéressent trop peu aux conjoints des adolescentes, d'autant plus qu'ils restent majoritairement absents.

\section{Typologie de mères adolescentes.}

Afin d'affiner notre analyse, une typologie des femmes a été créée distinguant trois profils d’adolescentes. Les modalités décrites dans le tableau 1 permettent de spécifier les différences entre les profils de jeunes femmes.

Le profil A correspond à 66 femmes essentiellement âgées de 12 à 15 ans. Ces adolescentes sont très largement déscolarisées (91\%), leur revenu propre ou leur activité professionnelle demeurent souvent inconnus, de même, elles déclarent rarement une protection sociale. Cependant leur situation conjugale et les rapports entretenus avec la famille apparaissent positifs : souvent en couple (73 \%) ou déjà mariées (42 \%), leur conjoint et leurs proches sont présents autour d'elles. Essentiellement originaires d’Europe de l’Est et plus précisément de Roumanie, ces adolescentes appartiennent à la communauté des gens du voyage : ces jeunes filles vivent principalement en caravane pendant la grossesse et après leur accouchement. Elles déclarent, presque unanimement, ne pas avoir utilisé de moyens de contraception avant d'être enceintes et sont plutôt réticentes à la proposition d'en prendre un après la naissance. Leur suivi de grossesse est très souvent inexistant ou chaotique : elles n'ont eu que peu de contacts avec l'équipe obstétricale. Les consultations obstétricales et les échographies sont très insuffisantes, tardives et il y a rarement de suivi précoce. Ces adolescentes donnent naissance à leur enfant très souvent par voie basse naturellement (86 \%), généralement sans aucun analgésique (notamment parce qu'elles n’ont pas consulté l'anesthésiste avant l'accouchement). Seulement 54 \% d'entre elles ont donné naissance à leur enfant au-delà des 37 semaines d'aménorrhée. 


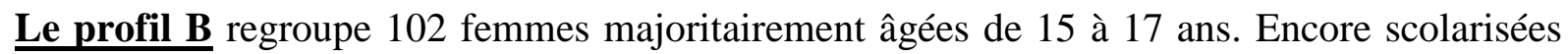
pour une large majorité (72\%), ces jeunes femmes ont un niveau secondaire et souhaitent souvent reprendre leurs études après la naissance de leur enfant. Elles déclarent être célibataires à 79 \% et le père de leur enfant est peu présent ou investi dans la grossesse. De même, les reconnaissances anticipées sont très rares pour leurs enfants. Ces adolescentes sont originaires plus souvent de France métropolitaine (44\%), vivent encore chez leurs parents à $77 \%$, mais nombreuses sont celles qui sont aussi hébergées (20 \%), notamment dans des structures d'accueil type foyer de l'Aide Sociale à l'Enfance (14\%). Elles ne déclarent presque aucune ressource financière propre mais disposent d'une protection sociale. neuf adolescentes sur dix ont déclaré ne pas avoir désiré la grossesse actuelle mais l’ont fait suivre convenablement : le nombre de consultations et d'échographies semble répondre aux exigences légales. De même, 74 \% d’entre elles ont déclaré avoir été prises en charge avant leur entrée à la maternité de l’hôpital (notamment par le service de PMI). Elles sont souvent en contact avec les professionnels de santé. Elles ont bénéficié à 57 \% de l’entretien prénatal précoce du $4^{\mathrm{e}}$ mois et $31 \%$ d'entre elles ont participé aux cours de préparation à l'accouchement. 77 \% de ces jeunes mères donneront naissance à un enfant à terme.

Enfin, le profil C correspond à 52 femmes en majorité âgées de 17 ans. Seul un quart d'entre elles est encore à l'école au moment du suivi de grossesse et $6 \%$ travaillent déjà. Majoritairement d'origine sub-saharienne (40\%), ces adolescentes sont en couple à $67 \%$. Elles vivent presque majoritairement hébergées (48 \%) au sein de leur famille ou chez des amis. $23 \%$ vivent avec leur partenaire dans un logement qui leur est propre. Notons un pourcentage non négligeable d'adolescentes vivant à la rue ou dans un squat (10 \%). Le père de l'enfant occupe souvent un emploi (54\%) et participe financièrement au bien-être du couple. De même, il s'investit plus souvent dans la grossesse et la venue de l'enfant. Les grossesses sont un peu plus désirées dans ce groupe de jeunes femmes (36 \%). La surveillance 
de la grossesse paraît convenable avec un nombre de consultations et d'échographies à peu près satisfaisant. De même, elles sont nombreuses à déclarer un suivi précoce de grossesse avant l'entrée en maternité (notamment en PMI) et un contact régulier avec les équipes soignantes (71 \% d'entre elles ont vu au moins une fois un psychologue). Leur grossesse est menée à son terme dans $83 \%$ des cas.

\section{Caractéristiques des divers types.}

- L’analyse de ces 3 profils révèle des différences sociodémographiques notables : la variable de l’âge reste peu révélatrice mais les adolescentes du groupe $C$ sont légèrement plus âgées. De même, des différences d'origines géographiques apparaissent très distinctement. La situation conjugale de ces adolescentes est aussi très contrastée : ce sont les jeunes femmes du groupe A qui déclarent vivre en couple le plus souvent (73 \%) dont 43 \% déjà mariées, versus $3 \%$ dans le groupe $B$. La situation professionnelle révèle également des divergences importantes : Ce sont les adolescentes du groupe B qui sont encore scolarisées dans une large majorité (72 \%) par rapport aux autres jeunes mères (et notamment les adolescentes du groupe A massivement déscolarisées). Ce sont elles aussi qui vivent le plus souvent encore chez leurs parents au moment de leur grossesse, à l’inverse du groupe C dont un quart déclare vivre dans un logement personnel. Enfin la variable score de vulnérabilité révèle des disparités notables : alors que les jeunes filles du groupe A sont susceptibles d'être davantage exposées à divers facteurs de vulnérabilité, notamment parce que le suivi de leur grossesse est inexistant et leur situation sociale, professionnelle, familiale, médicale est extrêmement peu documentée, ce sont celles qui apparaissent être les moins vulnérables. Les adolescentes du groupe B sont celles qui apparaissent les plus vulnérables (66 \%), suivies de près par les jeunes filles du groupe C (65\%). Pour ces deux derniers profils, des violences répétées ont été observées pour presque $20 \%$ d'entre elles et $9 \%$ de ces jeunes femmes ont été mises à la porte à l'annonce 
de leur grossesse. Enfin, 12 \% des adolescentes du groupe $\mathrm{C}$ ont déjà vécu un placement antérieur.

- La majorité des adolescentes n’a pas fait usage de contraception avant la grossesse (66 \%), avec des différences significatives selon le profil social et 64 \% ont déclaré ne pas avoir prévu la grossesse. Ce sont les adolescentes du profil C qui sont les plus nombreuses à exprimer un désir d'enfant (36 \%). A l’inverse les jeunes mères pour lesquelles la grossesse n’a pas été planifiée ni désirée, sont significativement plus souvent seules, décrivant une défaillance de leur partenaire, et appartiennent au groupe du profil B (90\%) : elles sont généralement scolarisées. Ce sont également des jeunes femmes qui vivent encore chez leurs parents avec des ressources financières faibles ou inexistantes.

\section{Les prises en charges obstétricales.}

- A propos de la surveillance de la grossesse, une jeune fille sur deux a été prise en charge avant l'entrée à la maternité de l’hôpital. Parmi celles-ci, 35 \% ont été prises en charge par le service de $\mathrm{PMI}^{1}$, 10 \% par un médecin généraliste de ville, 9 \% par un autre hôpital et enfin 1 \% par un service social scolaire, avec cependant un taux de non réponse très important (45 \%). Cependant des différences persistent : les jeunes femmes du profil B et C sont bien plus nombreuses (73 \% et 69 \%) à engager un suivi précoce de leur grossesse que celles du profil A (seulement $11 \%$ ). Cette prise en charge précoce semble influencer la qualité du suivi de toute leur grossesse car elles ont eu un nombre de consultations obstétricales et d'échographies plus important. De même, elles ont été plus souvent en contact avec les professionnels de santé et sont davantage impliquées dans la préparation de la venue de leur enfant en participant plus souvent au cours de préparation à l'accouchement : les différences sont ici flagrantes si l'on compare le profil A avec les profils B et C. Enfin, cette prise en charge précoce est fortement corrélée au terme de la grossesse : les adolescentes ayant eu un

\footnotetext{
${ }^{1}$ Centre de $\mathrm{PMI}=$ Protection Maternelle et Infantile
} 
suivi précoce de leur grossesse avant l'entrée à l'hôpital sont significativement plus nombreuses que les autres à donner naissance à un enfant à terme.

Au niveau du suivi obstétrical, les adolescentes ont bénéficié de cinq consultations prénatales en moyenne (5,9- écart type 4,2) et de deux échographies en moyenne (2,36 - écart type 1,77) mais près de $47 \%$ en ont eu moins de trois. Ici, les femmes les moins bien suivies étaient les femmes appartenant au groupe A, puisque près d’une adolescente sur deux n’a bénéficié d'aucune consultation obstétricale. Au sein de notre étude rétrospective, seules $41 \%$ des jeunes filles ont bénéficié d’un entretien prénatal précoce, avec un net avantage pour les profils B et C. Presque la totalité des adolescentes appartenant au groupe A n’a pas bénéficié de l'entretien prénatal du $4^{\mathrm{e}}$ mois (98\%).

Discussion. Le recueil rétrospectif de données au sein du centre hospitalier de Saint Denis nous a permis d’avoir un aperçu du phénomène des maternités précoces dans ce département de l'Ile-de-France qui concentre le plus grand nombre de grossesses adolescentes. Sans pour autant affirmer traiter le sujet dans sa globalité, notamment parce que nos données recueillies reposent sur le déclaratif des adolescentes comme des professionnels de santé, cette première étude nous a permis de déterminer trois profils de jeunes femmes et d'évaluer la qualité du suivi de leur grossesse. Il est également important de signaler qu'une partie du suivi de grossesse de ces jeunes femmes peut nous avoir échappé, surtout s’il était chaotique (adolescentes du groupe A par exemple) et réalisé par plusieurs acteurs de santé. Ces éléments peuvent conduire à une sous-évaluation de la qualité effective du suivi entre les groupes. Par ailleurs, le contexte de la grossesse ainsi que son suivi vont dépendre fortement du temps que les soignants accordent à chaque femme ainsi que la qualité de la communication établie avec elle. De même, comme évoqué en méthode, la qualité des données recueillies s’est améliorée entre 2007 et 2011, suite à la mise en place d’une consultation spécialisée pour ces adolescentes. Il est donc probable qu'il existe des erreurs de classement entre différentes 
modalités d’une caractéristique étudiée. Enfin la minorité des adolescentes a été évaluée au moment du début de la prise en charge de la grossesse. Les jeunes femmes non suivies ou très tardivement, mineures en début de grossesse mais pas à la fin n'auront pas été incluses.

Nous avons vu que les adolescentes de notre étude rétrospective se répartissent notablement au sein des trois profils créés : les plus jeunes appartiennent au groupe A et les plus âgées au groupe C. Pour autant, la variable de l’âge n’a pas révélé dans nos traitements statistiques de réels impacts sur la qualité du suivi de grossesse. En effet, l'hypothèse selon laquelle des adolescentes âgées de 17 ans seraient plus aptes que les plus jeunes à surveiller correctement leur grossesse en raison d'une maturité supposée n’a pas été vérifiée dans cette étude. La littérature tend à démontrer que globalement les grossesses chez les mineures sont moins bien suivies que les grossesses adultes, sans distinction précise de l'âge de ces adolescentes. Nos données corroborent ces résultats et nous le soulignerons par la suite [14, 17]. D’autres éléments sociodémographiques semblent bien plus pertinents pour évaluer le suivi de grossesse. Effectivement, la variable de l’origine géographique s’est révélée ici très significative : les jeunes femmes concernées par ces grossesses précoces sont plus souvent originaires d'Europe de l'Est et d'Afrique sub-saharienne. Nous constatons que ces observations semblent correspondre aux statistiques fournies par l’INED (entre 2005 et 2010) qui décrivent une répartition mondiale des grossesses adolescentes assez différenciée avec une fécondité très élevée en Afrique subsaharienne. De même, en Europe, la Roumanie présente, chez les jeunes femmes, l'un des taux de fécondité les plus élevés de l’Union [18]. Dès lors, la question se pose d’une grossesse précoce qui pourrait répondre à un phénomène culturel. Ce constat a été largement repris par des sociologues mais aussi des médecins qui évoquent le mariage et la maternité précoces chez des jeunes filles mineures (d’origine étrangère très souvent) comme une réponse à une dimension culturelle importante. Le professeur Michel Uzan évoque alors le concept de « grossesses culturelles », qui s’avère ici très caractéristique : 
dans de nombreuses sociétés africaines, Est-européennes ou maghrébines, avoir un enfant est très valorisant. La grossesse est un rite de passage au cours duquel les jeunes filles deviennent des femmes adultes. Ces grossesses sont alors très souvent programmées, attendues par un couple ou une famille. Ainsi, la maternité précoce est très différente chez ces adolescentes puisqu'elle s'insère dans un cadre culturel, social et familial favorable à la naissance d'enfants chez des jeunes mères [19-21]. Ces observations ont été vérifiées au sein de notre étude : c’est notamment le cas des profils A et C. Ces adolescentes sont bien souvent déjà mariées ou en couple. Ces grossesses apparaissent alors plus souvent désirées, investies (notamment les adolescentes du profil C) et se déroulent globalement de façon simple. Dans son travail ethnographique sur des roms roumains vivant en Île-de-France, C. Knaff indique que les jeunes filles présentes sur le campement « choisissent leur mari » et se marient globalement vers 14-15 ans. Ce mariage, très important, doit perdurer toute la vie et ces femmes se doivent d'avoir beaucoup d'enfants. Compte tenu de l'âge précoce au mariage et de la faible couverture contraceptive, il est donc fréquemment observé des grossesses parmi les adolescentes roms [22].

Bien que les profils B et C se rejoignent sur ce sujet, nous verrons que leur suivi de grossesse est en tous points très différents. Il est donc tout à fait important de tenir compte du contexte culturel et social dans lequel la grossesse évolue [23].

Le taux de scolarisation nous a aussi interpellés : il est souvent évoqué un risque de déscolarisation important et une mauvaise intégration sociale chez les mères adolescentes. Nombre d'auteurs évoquent la survenue d'une grossesse à l'adolescence comme un risque d'exacerbation des difficultés socioéconomiques avec la déscolarisation en tête : notre étude tend à le confirmer puisque majoritairement les jeunes filles étudiées sont en dehors du circuit scolaire au moment de leur grossesse. Selon Faucher et al (2002), devenir mère pour une adolescente peut s’avérer être une stratégie d’adaptation pour échapper à une scolarité peu 
valorisante : cela pourrait être un moyen de valorisation puisqu'accéder à un statut de mère confère un statut social. Avoir un enfant leur octroierait une situation plus enviable d'autonomie et de respect par l'entourage [3]. Nous avons malheureusement peu d'information sur le taux de scolarisation après accouchement des jeunes femmes de notre étude et seule une minorité d'entre elles a fait part d'un projet professionnel ultérieur à l'équipe obstétricale. Ce phénomène est en lien avec le jeune âge de ces mères qui, comme beaucoup d'adolescents, vivent très souvent dans l'instantanéité, avec une difficulté à se projeter sur le long terme [24]. L’éviction scolaire risque alors d’aggraver le pronostic social ultérieur de la jeune femme et de son enfant par conséquent. Pour de nombreux auteurs, c’est un risque psychosocial important dont une menace d'inadaptation sociale et professionnelle, des difficultés de logement, une précarisation des conditions de vie, une absence de ressources financières propres [25-27].

Enfin, nos données montrent que ces adolescentes sont minoritaires à déclarer vivre dans un logement qui leur est propre (5\%) ce qui est habituel pour ce groupe d’âge [21, 24]. Par contre nous constatons une multiplication des situations d'hébergement (amis, familles, structures d’accueil) voire de jeunes femmes à la rue. Ces fragilités se sont vérifiées au travers de notre variable score qui révèle que plus de la moitié des jeunes filles de notre cohorte cumule au moins deux facteurs de vulnérabilité pendant la grossesse. Les plus concernées, rappelons-le, sont les adolescentes du profil B, qui loin de mener une grossesse «culturellement » encouragée et souhaitée sont celles qui apparaissent les plus vulnérables. Leur grossesse ne présente donc pas le même dessein : plus souvent accidentelles, survenant dans le cadre d'une sexualité mal ou non protégée, elle pourrait être le résultat d'une conduite à risque et vue comme une solution pour échapper à une scolarité ou un milieu familial perturbé. La naissance de l'enfant risque alors d'aggraver leur précarité et leur isolement [2, 3]. La question de la contraception a également été soulevée dans cette étude mais majoritairement 
les jeunes filles n'en ont pas fait usage avant leur grossesse. Les adolescentes du profil A sont celles qui paraissent les plus réticentes face à la contraception, même après la naissance de leur enfant : l'association Médecins du Monde estime que les femmes roms sont généralement peu demandeuse de moyen de contraception même si cela tend à changer (notamment parce que l'information autour de la contraception comme outil de régulation des naissances est fortement déficiente en Roumanie). L’enquête, conduite en 1999 par questionnaire quantitatif auprès de 78 personnes roms de nationalité roumaine vivant sur trois terrains à Gennevilliers, permet d’apporter des précisions. Parmi les femmes interrogées, 97 \% ont déclaré n’avoir jamais eu recours à un moyen contraceptif. L’âge moyen à la naissance du premier enfant était de 17 ans. Les femmes ont déclaré en moyenne 6,2 grossesses. Le recours à l'IVG (autorisé depuis 1957 en Roumanie) constitue alors le principal mode de régulation des naissances ce qui pose de larges problèmes pour les autorités de santé [22].

Bien que la problématique paraisse différente pour les profils B et C, les explications sur cette absence de moyens de contraception ou sa mauvaise utilisation sont multiples et tendent à mettre en évidence un paradoxe dérangeant : en France où la couverture contraceptive est la plus élevée au monde, où l’âge moyen au premier enfant approche des trente ans, le nombre d'IVG chez les mineures reste toujours très élevé [28]. Et c'est bien là que se situe la contradiction: les adolescentes françaises ne sont pas des contraceptives assidues. Des problèmes d'observance, d'utilisation, de coût ou la crainte d'un rendez-vous gynécologique avec tout ce que cela suggère (peur de l'examen ou d'un secret professionnel non respecté) peuvent expliquer ce constat [29]. De même, nombreuses sont celles qui associent la prise de la pilule à de nombreux préjugées obsolètes comme la prise de poids ou la stérilité. Enfin, bien souvent les histoires amoureuses sont, à cet âge, de courte durée. L'utilisation de la pilule n’est pas alors forcément adéquate. Ces explications omettent la dimension inconsciente du désir de grossesses chez certaines jeunes femmes pour lesquelles la maternité offre une 
échappée, la promesse valorisante d'un statut et d'une identité sociale, une tentative de réparation d'une enfance tumultueuse [26]. Le principe de réalité va alors agir comme un couperet dans leurs illusions prénatales. Être enceinte peut être aussi un moyen de tester sa fertilité, de vérifier l'intégralité de son corps, de s’assurer que ce dernier fonctionne correctement [29].

Le suivi de grossesse des adolescentes de notre étude et le nombre de consultations prénatales et d’échographies réalisées restent globalement inférieurs aux données nationales françaises. Dans l'enquête nationale périnatale, plus de neuf femmes sur dix ont bénéficié d’au moins sept visites prénatales en 2010 [30]. Au sein de notre étude rétrospective, le nombre de visites n’est que de cinq consultations prénatales en moyenne. Il convient cependant de modérer ces données en fonction de la durée de gestation et du moment de la déclaration de grossesse : les femmes accouchant prématurément consultent moins en raison d’une grossesse plus courte et quand la déclaration est tardive, les premiers examens n’ont pas pu avoir lieu. Ainsi, au sein de notre étude rétrospective, $43 \%$ des déclarations de grossesse sont faites au cours du $2^{\mathrm{e}}$ trimestre. En ce qui concerne le nombre d’échographies, les données nationales estiment qu'en France en 2010, les femmes ont eu recours à un peu plus de cinq échographies en moyenne, alors qu'au sein de notre étude, les jeunes femmes ont eu deux échographies en moyenne. De même, la surveillance de grossesse débute majoritairement tardivement, autour du $2^{\mathrm{e}}$ trimestre. Cependant, la typologie laisse apparaître des différences caractéristiques qui ont un impact majeur sur le suivi de la grossesse. Notre étude a montré que le mauvais suivi des grossesses, ou un suivi insuffisant reste inhérent aux jeunes filles appartenant au groupe A. Nous avons vu que la grossesse chez une adolescente est culturellement valorisée dans ce groupe et s’inscrit dans le maintien d’une identité collective. Pour autant, le suivi de grossesse reste insuffisant et tardif, bien qu'il ne semble pas être propre aux mères adolescentes mais bien aux femmes enceintes de cette origine en général. Le constat, commun aux acteurs 
intervenant auprès des roms, est la quasi-absence de suivi des femmes roms pendant leur grossesse. Selon les données de Médecins du Monde recueillies auprès des femmes roms en 2007, seule une femme sur dix était suivie durant sa grossesse. Il n’est ainsi pas rare que des femmes viennent aux urgences des maternités le jour de l'accouchement, sans consultations prénatales préalables. Cette enquête révèle également «une tendance des femmes à ne pas percevoir la grossesse comme nécessitant une médicalisation »[22]. Dès lors, les risques pour le nouveau-né d'une prise en charge tardive sont notables et plus fréquents dans ce groupe de jeunes femmes. Méfiance ou souhait volontaire d'éviter les circuits de prise en charge de la grossesse, leurs motivations sont peu connues tant leurs contacts avec les équipes soignantes sont rares. Ce même rapport met en relief différents éléments explicatifs : l’accès aux soins est souvent tardif en raison de l'absence d'ouverture de droit et de l'irrégularité de leur situation sur le territoire. La logique de l'urgence est très différence au sein de la population roms qui va parfois privilégier d'autres situations du quotidien à celui du suivi de grossesse, surtout si tout paraît normal (le logement, trouver des ressources ...). L’absence de stabilité des lieux de vie propre aux populations roms constitue également une rupture dans le processus du suivi de ces femmes et fragilise fortement les liens établis avec les équipes soignantes. Enfin, leur rapport au corps est particulier : le recours au soin semble se faire « lorsque le problème de santé constitue un handicap dans le quotidien [...] les roms ne sont à l'écoute de leur corp que lorsque ce dernier crie une souffrance intolérable » [22].

A l'inverse, les adolescentes du groupe B et C ont eu un suivi de grossesse de meilleure qualité et l'état de santé de leur enfant est moins préoccupant. Pour les jeunes filles du groupe B, cette meilleure prise en charge pourrait s'expliquer par le fait qu'elles sont encore scolarisées massivement (72 \%), souffrant ainsi d'une exclusion sociale moins forte que leurs congénères, les rendant davantage informées sur les offres de soin et les circuits de santé. Quant aux jeunes filles du groupe C, elles sont un peu plus nombreuses que les autres à 
déclarer désirer leur grossesse, elles sont majoritairement en couple et leur investissement est plus notable avec une surveillance de grossesse engagée avant l'entrée en maternité. Autant d'éléments qui influencent la qualité d’un suivi de grossesse et le pronostic vital de l'enfant. Par ailleurs, comme évoqué antérieurement, nous n’avons pas développé la question de la consommation de substances ni avant ni pendant la grossesse, bien que notre questionnaire contenait ces items. Les dossiers obstétriques consultés ne renseignaient pas suffisamment les usages de substances pour qu'ils soient exploitables. Moins d’1\% des jeunes filles ont déclaré consommer de l'alcool, du cannabis ou des drogues pendant leur grossesse. Le tabac reste le plus consommé, occasionnellement ou tous les jours pour 19,5 \% des adolescentes en début de grossesse (pourcentage non varié en fin de grossesse) et concerne essentiellement les jeunes femmes du profil A. Les effets du tabac sur la prématurité, le retard de croissance intra utéro mais aussi le risque de mort subite du nourrisson sont aujourd'hui extrêmement bien documentés. Or cette variable s’est avérée très mal renseignée dans notre questionnaire [31]. D’autre part, nous avons vu qu'une prise en charge précoce influence notablement la qualité du suivi de grossesse et le pronostic vital de l'enfant à naître. Depuis deux ans déjà, l’hôpital Delafontaine, a mis en place une consultation spécialisée et exclusivement réservée à la surveillance de la grossesse chez des jeunes femmes mineures. Une équipe pluridisciplinaire, constituée de sage-femmes, psychologues, assistantes sociales, puéricultrices, propose un accompagnement précoce et continue pour ces adolescentes, notamment caractérisé par la mise en contact de la jeune mère avec divers acteurs sociaux et de santé. Ainsi, une consultation avec une assistante sociale et une psychologue est proposée obligatoirement à toute mineure enceinte au cours de sa grossesse. L’entretien prénatal précoce est aussi un outil qui trouve toute sa place dans l'accompagnement du suivi de grossesse chez une jeune mineure, tout en tenant compte de son histoire de vie. Proposé à toutes les femmes enceintes, au cours du 4ème mois de grossesse, il doit permettre de préparer avec elles les meilleures 
conditions possibles de la venue au monde de leur enfant, notamment en repérant la vulnérabilité de certaines populations dites «à risque » que sont, entre autres, les mères adolescentes. Il convient d'insister sur l'extrême diversité de ces grossesses adolescentes et sur l'importance de l'entourage et du contexte culturel dans lequel l'adolescente évolue pour préparer au mieux avec elle la naissance de son enfant. Pour autant, il ne faut pas perdre de vue que ces adolescentes se font suivre tardivement et il ne faut donc pas réduire cet entretien à une limite temporelle fixe mais plutôt permettre aux soignants comme à l'adolescente de pouvoir s’exprimer lorsque le moment sera le plus juste. De même, un partenariat avec les équipes de réseaux (PMI, planning familial, centre hospitalier etc.) semble renforcer l'étayage nécessaire à une meilleure surveillance de grossesse chez une très jeune femme. La qualité du suivi de grossesse est un facteur important à analyser avant d'étudier les éventuelles complications obstétricales chez les mères adolescentes. L'exploitation de cette enquête va donc être poursuivie à ces fins, pour examiner l'influence de ces profils de jeunes mères sur les caractéristiques de leurs nouveau-nés.

Remerciements. Ce travail bénéficie d’un soutien financier de la région Ile-de-France, sous forme d'une allocation doctorale pour une durée de trois années. 
Tableau 1 : caractéristiques sociodémographiques et contexte de la survenue de la grossesse/ Socio démographic characteristics and context of the pregnancy

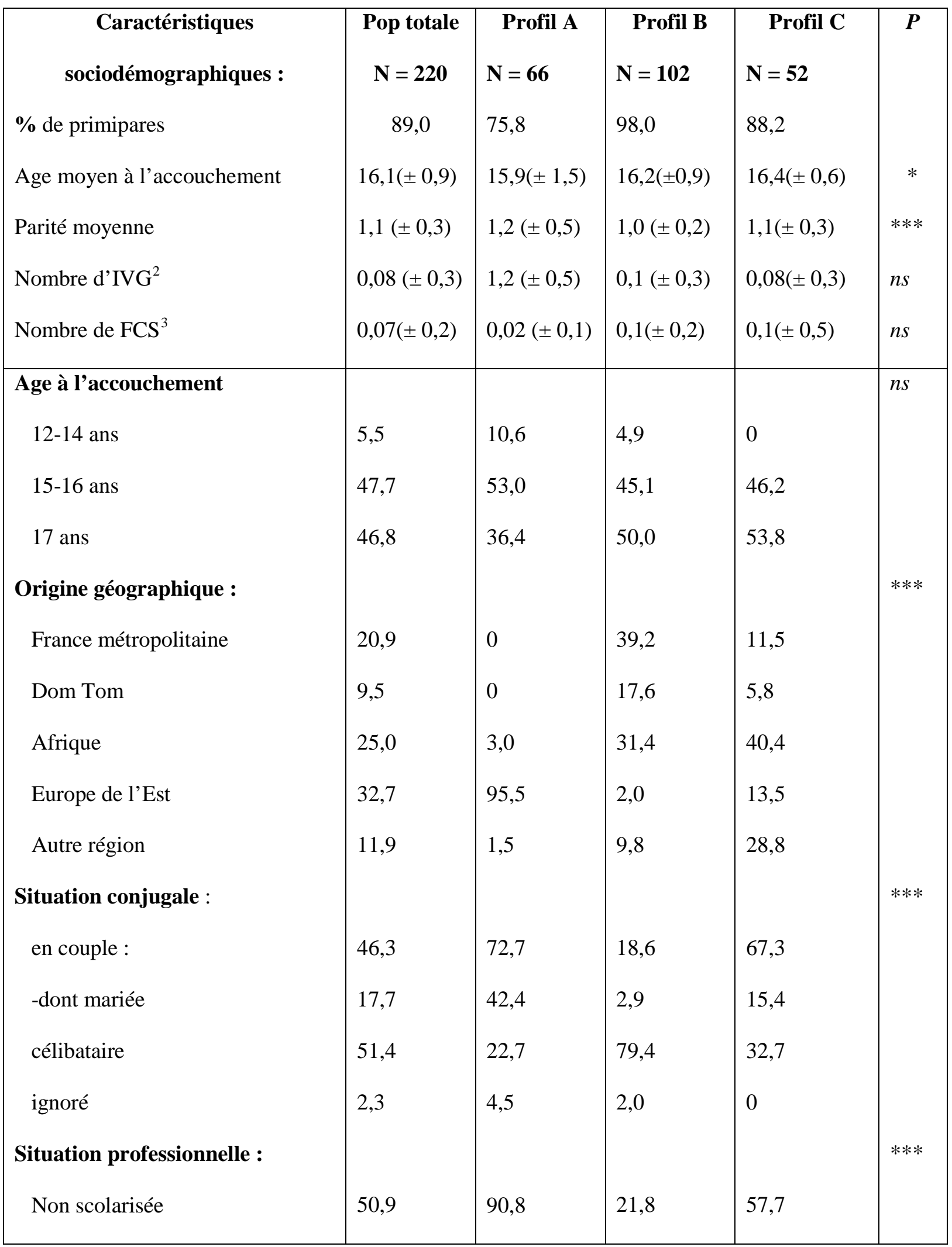

${ }^{2}$ IVG : interruption volontaire de grossesse.

${ }^{3}$ FCS : Fausse couche spontanée. 


\begin{tabular}{|c|c|c|c|c|c|}
\hline Scolarisée & 39,4 & 0 & 72,3 & 25,0 & \multirow[b]{4}{*}{$* * *$} \\
\hline Sans activité & 7,3 & 7,7 & 4,0 & 11,5 & \\
\hline Travaille & 2,4 & 1,5 & 2,0 & 5,8 & \\
\hline \multicolumn{2}{|l|}{ Logement durant la grossesse : } & & & & \\
\hline Chez ses parents : & 69,1 & 95,5 & 77,5 & 19,2 & \\
\hline -dont caravane (gens du voyage) & 28,2 & 93,9 & 0 & 0 & \\
\hline Hébergée : & 20,9 & 1,5 & 19,6 & 48,1 & \\
\hline -dont structures & 6,8 & 0 & 14,7 & 0 & \\
\hline -dont famille, amis & 14,1 & 1,5 & 4,9 & 48,1 & \\
\hline Personnel & 5,5 & 0 & 0 & 23,1 & \\
\hline Rue, squatt & 2,7 & 1,5 & 0 & 9,6 & \\
\hline Ignoré & 1,8 & 1,5 & 2,9 & 0 & \\
\hline Variable vulnérabilité : & & & & & $* * *$ \\
\hline Non vulnérable & 40,7 & 57,4 & 33,7 & 35,4 & \\
\hline Vulnérable & 59,3 & 42,6 & 66,3 & 64,6 & \\
\hline Grossesse désirée : & & & & & $* * *$ \\
\hline Oui & 14,5 & 9,1 & 6,9 & 36,5 & \\
\hline Non & 63,6 & 28,8 & 90,2 & 55,8 & \\
\hline Ignoré & 21,8 & 62,1 & 2,9 & 7,7 & \\
\hline Conflits familiaux : * & & & & & \\
\hline Mise à la porte & 6,8 & 1,5 & 8,8 & 9,6 & ns \\
\hline Violences & 14,1 & 3,0 & 19,6 & 17,3 & $* * *$ \\
\hline -dont viols & 3,2 & 0 & 4,9 & 3,8 & $* * *$ \\
\hline Placements antérieurs & 16,2 & 0 & 28,7 & 11,8 & $* * *$ \\
\hline
\end{tabular}

*Totaux différents de 100 \% car variables multiples. 
Tableau 2 : Le suivi prénatal/ Prenatal Care.

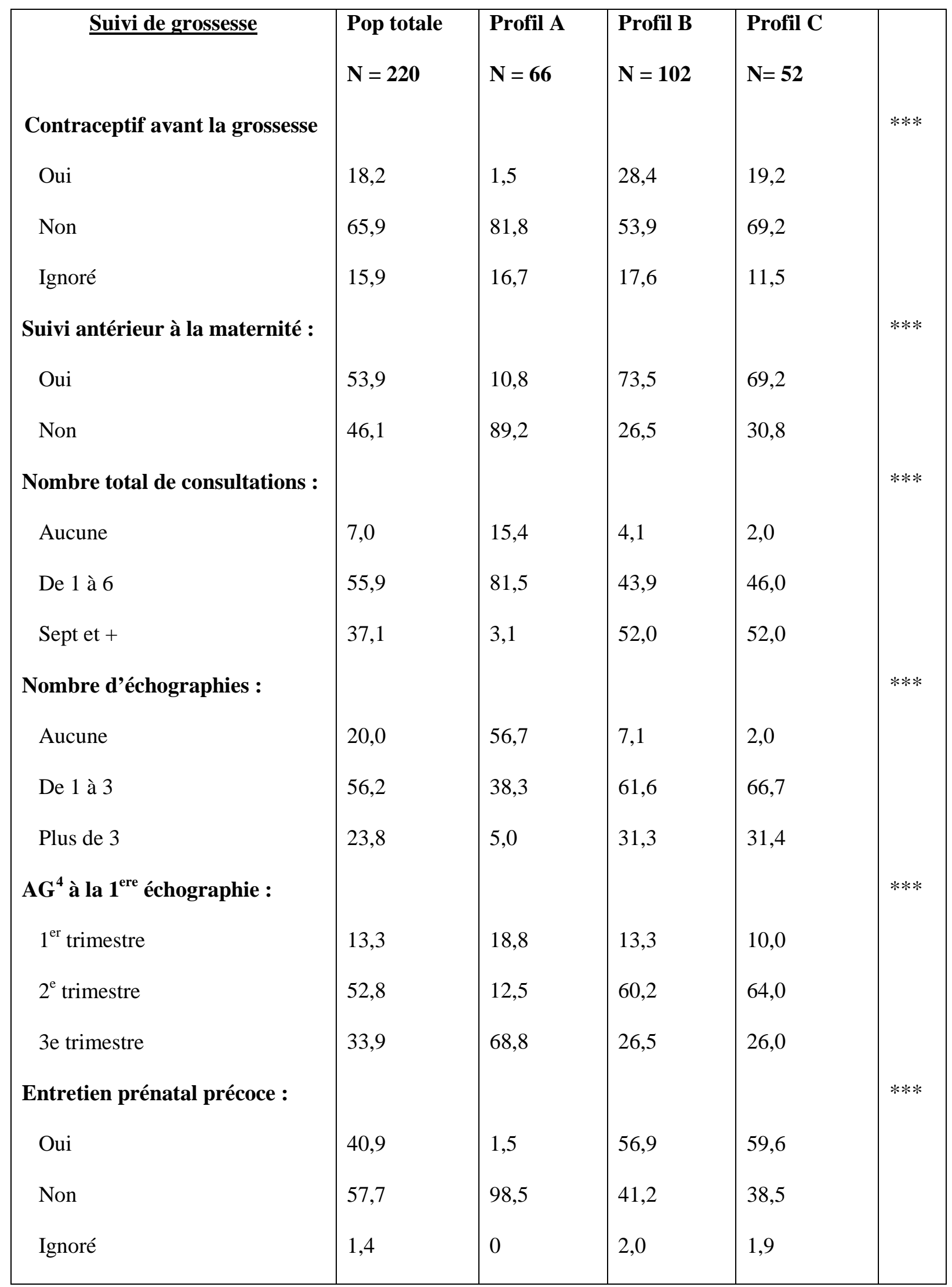

${ }^{4} \mathrm{AG}$ : Age gestationnel. 


\begin{tabular}{|c|c|c|c|c|c|}
\hline $\begin{array}{l}\text { Prise de contact avec une } \\
\text { psychologue }\end{array}$ & & & & & $* * *$ \\
\hline Oui & 60,9 & 15,2 & 85,3 & 71,2 & \\
\hline Non & 38,2 & 84,8 & 13,7 & 26,9 & \\
\hline Ignoré & 0,9 & 0 & 1,0 & 1,9 & \\
\hline \multicolumn{6}{|l|}{ Accouchement } \\
\hline AG : Moyenne en $\mathrm{SA}^{5}$ & $37,8( \pm 4,1)$ & $37,7( \pm 3)$ & $37,6( \pm 4,8)$ & $38,1( \pm 3,5)$ & $n s$ \\
\hline $\mathrm{AG}<37 \mathrm{SA}($ en $\%)$ & 71,4 & 54,5 & 76,5 & 82,7 & $* * *$ \\
\hline Voie d'accouchement : & & & & & $* *$ \\
\hline Voie basse naturelle & 71,5 & 85,7 & 71,0 & 54,9 & \\
\hline Voie basse instrumentale & 17,3 & 9,5 & 19,0 & 23,5 & \\
\hline Césarienne & 11,2 & 4,8 & 10,0 & 21,6 & \\
\hline Analgésie & & & & & $* * *$ \\
\hline Péridural ou rachi anesthésie & 56,7 & 20,6 & 70,2 & 74,5 & \\
\hline Anesthésie générale & 4,7 & 4,8 & 5,0 & 3,9 & \\
\hline Rien & 38,6 & 74,6 & 24,8 & 21,6 & \\
\hline
\end{tabular}

ns : non significatif ; ${ }^{*} \mathrm{p}<0,05 ;{ }^{* *} \mathrm{p}<0,01 ;{ }^{* * *} \mathrm{p}<0,001$.

${ }^{5}$ SA : Semaine d'aménorrhée. 


\section{Références.}

1. Aubin, C. and D. Jourdain Menninger, Rapport Igas : La prévention des grossesses non désirées : contraception et contraception d'urgence. 2009, Inspection générale des affaires sociales: Paris.

2. Filou, A., La maternité à l'adolescence : une prise en charge spécifique ? Etude comparative 1998-2010 sur une population d'adolescentes ayant menées leur grossesse à terme dans la même maternité., ed. E.U. Europeennes. 2011, Paris.

3. Nisand, I. and L. Toulemon, Pour une meilleure prévention de l'IVG chez les mineures. 2006, Haut conseil de la population et de la famille: Paris.

4. Dugeny, F., Fécondité soutenue en Ile-de-France. Note rapide Population-Mode de vie, 2008. 443.

5. Galaup, K., Parcours de santé et interruption volontaire de grossesse, A.R.d. Santé, Editor. 2012, ARS. p. 22-28.

6. http://www.perinat-ars-idf.org/, flux périnatalité. 2010: Paris.

7. Bajos, N., A. Guillaume, and O. Kontula, Le comportement des jeunes européens face à la santé génésique. Etudes démographiques, 2004. 1(42): p. 149.

8. Daguerre, A., Les grossesses adolescentes en France et en Grande-Bretagne. Un phénomène dérangeant pour les pouvoirs

publics. Informations sociales, 2010(157): p. 96-102.

9. Pison, G., Les maternités précoces en recul dans le monde. Population \& sociétés. Bulletin mensuel d'information de l'Institut national d'études démographiques., 2012(490): p. 1-4. 
10. S Khashan, A., P. N Baker, and L. C Kenny, Preterm birth and reduced birthweight in first and second teenage pregnancies: a register-based cohort study. BMC Pregnancy and Childbirth 2010. 10(36): p. 10.

11. Berrewaerts, J. and F. Noirhomme-Renard, Les grossesses à l'adolescence : quels sont les facteurs explicatifs identifiés dans la litterature ?, E.p.l. santé, Editor. 2006, UCL RESO, faculté de médecine de Louvain: Louvain. p. 36.

12. Godeau, E., et al., Facteurs associés à une initiation sexuelle précoce chez les filles : données françaises de l'enquête internationale Health Behaviour in School-aged Children (HBSC)/OMS. Gynécologie Obstétrique \& Fertilité, 2008. 36(2): p. 176-182.

13. OMS, Adolescentes enceintes : apporter une promesse d'espoir dans le monde entier, UNFPA, Editor. 2007: Suisse. p. 26.

14. Scheidegger, S. and A. Vilain, Disparités sociales et surveillance de grossesse. Etudes et résultats, 2007(552): p. 1-8.

15. Moutquin, J.-M., Classification and heterogeneity of preterm birth. BJOG: An International Journal of Obstetrics \& Gynaecology, 2003. 110(20): p. 30-33.

16. Insee, Bilan démographique 2009. Pages de profils, Nord Pas-de-calais., 2010. 72: p. 4.

17. Charreire, H., Impact conjugué des déterminants sociaux et géographiques sur le suivi de la grossesse en milieu urbanisé., in Congrès national des Observatoires régionaux de la santé. 2008: Marseille.

18. Pison, G., Communication à la 3e journée humanitaire sur la santé des femmes., G.s. frontière, Editor. 2011: Paris. 
19. Dedecker, F., et al., Etude des facteurs de risques obstétricaux dans le suivi de 365 grossesses primipares adolescentes à l'Île des la Réunion. J Gynecol Obstet Biol Reprod, 2005. 34: p. 694-701.

20. Uzan, M., Rapport sur la prévention et la prise en charge des grossesses des adolescentes. 1998, Université Paris XIII, Assistance Publique - Hôpitaux de Paris, INSERM U 361: Bondy. p. 31.

21. Faucher, P., S. Dappe, and P. Madelenat, Maternité à l'adolescence : analyse obstétricale et revue de l'influence des facteurs culturels, socio-économique et psychologiques à partir d'une étude rétrospective de 62 dossiers. Gynécologie Obstétrique \& Fertilité., 2002. 30: p. 944-952.

22. Halfen, S., Situation sanitaire et sociale des "Rroms migrants » en Île-de-France. Rapport de l'Observatoire régional de santé d'Île-de-France. 2012, ORS île-deFrance: Paris. p. 146.

23. Hamada, H., Grossesse et accouchement chez l'adolescente : caractéristiques et profil. J Gynecol Obstet Biol Reprod, 2004. 33: p. 5.

24. Le Van, C., Les grossesses à l'adolescence. Normes sociales, réalités vécues., ed. L'Harmattan. 1998, Paris. 204.

25. Mottrie, C., L. de Coster, and I. Duret, Devenir mère : transformations des liens et des lieux familiaux lors des grossesses survenant à l'adolescence. Cahiers critiques de thérapie familiale et de pratiques de réseaux, 2006. 37(2): p. 121-137.

26. Mouras, J.-P., Être mère à l'adolescence. Figures de la psychanalyse, 2004. 9(1): p. 97-101. 
27. Verdure, F., et al., Connaissances, besoins et attentes des adolescents en éducation sexuelle et affective. Étude réalisée auprès d'adolescents de classes de troisième. Archives de Pédiatrie, 2010. 17(3): p. 219-225.

28. Alvin, P., Contraception chez l'adolescente: le grand paradoxe. Archives de Pédiatrie, 2006. 13(4): p. 329-332.

29. Bajos, N. and M. Ferrand, De la contraception à l'avortement. Sociologie des grossesses non prévues. Questions en santé publique, ed. Inserm. 2002, Paris.

30. Brocas, A.-M., La situation périnatale en France en 2010. Etudes et résultats. . 2011, Dress: Paris.

31. Delcroix, M.-H., C. Gomez, and B. Dautzenberg, Grossesse et tabac : les leçons de trois études pour améliorer les pratiques professionnelles. La revue Sage-Femme, 2012. 11: p. 81-86. 